\title{
Path Planning and Visualizing for Maintenance Simulation of Nuclear Power Plant
}

\author{
http://dx.doi.org/10.3991/ijoe.v9i6.3008 \\ Guangwei Yan, Miao Li \\ North China Electric Power University, Beijing, China
}

\begin{abstract}
During the refueling period of a nuclear power plant, the plant needs to be checked and maintained carefully. The maintenance procedure is dangerous to the maintenance personnel because of the radiation in the plant. To train maintenance personnel and decrease the radiation dose received by them, we designed a maintenance simulation system of nuclear power plant by means of virtual reality technology. A virtual nuclear power plant scene which includes equipments, pipes, rooms and virtual maintenance people is built. The activities of the virtual maintenance person are simulated through the threedimensional (3D) animation technology. The radiation fields in the plant are showed through a color plane rendering method. To find the optimal maintenance path that has the smallest cumulative radiation dose in the radiation environment, an improved ant colony algorithm is proposed. The designed system can help to train the maintenance personnel and to increase the safety of the maintenance procedure of the nuclear power plant.
\end{abstract}

Index Terms-Nuclear Power Plant, Virtual Reality, Maintenance Simulation, Path Planning, Ant colony Algorithm

\section{INTRODUCTION}

After the grave nuclear accident occurred in the Fukushima nuclear power plant, Japan, the safety of nuclear power plants caused more and more debate. Most of the nuclear accidents are caused by misoperation of operators, so it is very important to increase the operation skill of staff in the nuclear power stations. Some simulation systems of nuclear power stations have been designed to help to train staff in nuclear power stations. Ohga et al. [1] designed a system which can calculate and visualize the radiation field, and can help the maintenance personnel. Lamela et al. [2] and Ródenas et al. [3] designed virtual reality systems to help to train nuclear power plant operators. Vermeersch et al. [4] designed a planning tool to decrease the radiation dose received by operators. Some simulation systems that can be used in decommissioning procedure of nuclear facilities were designed [5]-[8]. Kim et al. [9] proposed a calculating method to predict the radiation dose in nuclear power plants. Yan et al. [10] designed a virtual inspection system of nuclear power stations.

During the refueling period of a nuclear power station, a lot of equipments need to be checked and maintained. The proficient operation ability of the maintenance personnel can decrease the hidden troubles existing in equipments. And the maintenance personnel need to be protected and their working time should be decreased because of the radiation in some places of the plant. To

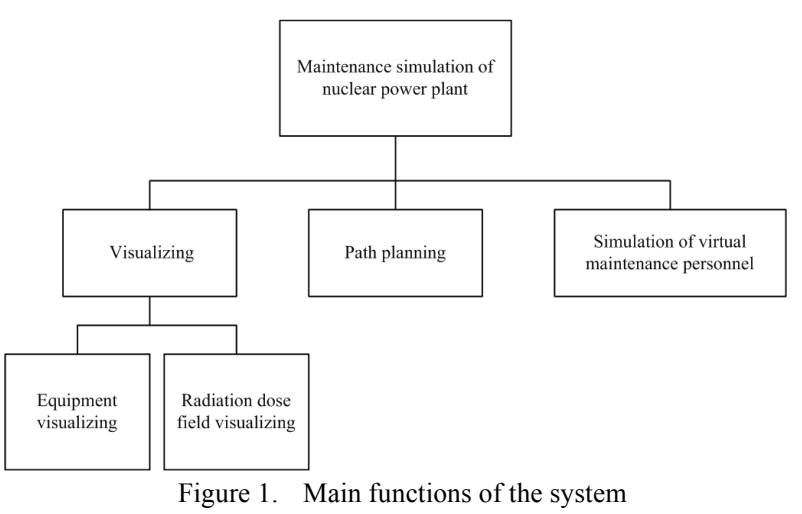

train the maintenance personnel and decrease the radiation dose received by them, we designed a maintenance simulation system of nuclear power plant. To construct a vivid maintenance simulation environment, we built the 3D nuclear power station models by the Multigen Creator tool [11]. And these nuclear power station models and the color radiation field are organized and rendered based on the Vega graphics engine [12]. A virtual maintenance personnel is modeled and it can do various operations in the virtual nuclear power plant scene. Under the restriction of minimum cumulative dose received by the maintenance personnel, an ant colony planning algorithm [13]-[16] is used in our system to calculate the optimal maintenance path.

\section{SYSTEM FRAMEWORK}

The main functions of the simulation system include: $3 \mathrm{D}$ roaming of the nuclear power plant, visualizing of the radiation dose fields, planning and visualizing of the maintenance path, activity simulation of the virtual maintenance personnel. The function diagram is showed in Fig.1.

There are various equipments and objects in a nuclear power station. First, they are modeled through 3D modeling tools. Then, these 3D models are organized into a 3D scene. Users can roam in the virtual scene and do various operations.

To show the invisible radiation field in the $3 \mathrm{D}$ scene, a radiation dose field rendering method is designed. It uses different colors to represent different radiation dose values.

By means of virtual person modeling technology and animation technology, various activities of the virtual maintenance personnel is simulated.

After a maintenance task is assigned, the ant colony planning algorithm is used to find an optimal maintenance 
path. This path has the minimum cumulative radiation dose value compared with other maintenance paths.

\section{Maintenance Path PlanNing}

To do the optimal path planning in the radiation environment, firstly, the environment is divided into uniform grids in horizontal direction. The size of each grid is denoted as step*step and the value range of the parameter step is set as $0.2 \mathrm{~m} \sim 1.0 \mathrm{~m}$. The whole grid space is denoted as $S\left(N_{x}, N_{y}\right)$.The parameter $N_{x}$ means the grid number in $x$ axis direction, and the parameter $N_{y}$ means the grid number in $y$ axis direction. The radiation dose rate at the center of each grid is calculated by linear interpolating to the known radiation dose rate in the environment space. We assume the virtual maintenance

$$
\operatorname{dose}(i, j)=\left\{\begin{array}{l}
(\operatorname{rdrate}(i)+\operatorname{rdrate}(j)) \times\left(\frac{0.5 \times \text { step }}{v}\right) \\
(\operatorname{rdrate}(i)+\operatorname{rdrate}(j)) \times\left(\frac{0.5 \times \sqrt{2} \times \text { step }}{v}\right)
\end{array}\right.
$$

if $i, j$ are horizonal or vertical neighboring grids

if $i, j$ are neighboring grids in diagnal direction

The parameters $\operatorname{rdrate}(i)$ and $\operatorname{rdrate}(j)$ denote the radiation dose rate of the grid $i$ and $j$ separately.

\section{B. Path planning}

We use the ant colony algorithm to calculate the best maintenance path between the beginning point $V_{\mathrm{o}}$ and the target point $V_{\mathrm{t}}$ of a maintenance task in the radiation environment. The calculation procedure is as follows.

\section{1) Initializing of the ant colony algorithm's parameters}

The ant colony algorithm's parameters include $\alpha, \beta, \rho$, $m, Q$, iter $\max$, the pheromone trail matrix and the paths table of the ants' passed, etc. Where $\alpha$ denotes the importance degree of the pheromone trail; $\beta$ denotes the importance degree of the heuristic function; $\rho$ is the volatile factor of the pheromone; $Q$ denotes the increase degree of the pheromone; iter $_{\max }$ denotes the maximum iteration calculating amount the algorithm allowed.

\section{2) Moving control of the ants}

The $V_{\mathrm{o}}$ and $V_{\mathrm{t}}$ grids are set $m$ ants separately. For the ant $k$, its current grid $i$ 's accessible grid set is constructed as (2).

$$
\text { allow }_{i}^{k}=N_{i}-P_{k}
$$

The set $N_{i}$ is the grid $i$ 's neighbor grids, and the set $P_{k}$ is the grids the ant $k$ has passed.

The ant $k$ selects the target grid in allow $_{i}^{k}$ and moves to it according to (3).

$$
p_{i j}^{k}=\left\{\begin{array}{cl}
\frac{\tau_{i j}^{\alpha} \operatorname{dose}(i, j)^{-\beta}}{\sum_{g \in \text { allow }_{i}^{k}} \tau_{i g}^{\alpha} \operatorname{dose}(i, g)^{-\beta}}, & j \in \text { allow }_{i}^{k} \\
0, & \text { else }
\end{array}\right.
$$

The variable $p_{i j}^{k}$ denotes the probability moving from the grid $i$ to the grid $j$ to the ant $k$. And $\tau_{i j}$ is the pheromone trail concentration from the grid $i$ to the grid $j$. Here $\operatorname{dose}(i, j)^{-\beta}$ acts as the heuristic function when the ant moves from the grid $i$ to the grid $j$.

\section{3) Death setting of the ants}

Every ant selects its next grid according to (3) and this grid number is stored in its passed path record. After every ant has moved to new girds, the system will judge whether the ant should be dead or not. The death rules of the ants are described as follows. personnel locate at the center of a grid when they move or operate in the radiation environment. Below, to simplify the description, we use the term "grid" to represent the center point of the grid. We denote the maintenance personnel walking speed by $v$.

\section{A. Calculation of received radiation dose when passing through the grids}

To calculate the accumulative radiation dose received by the maintenance personnel in a maintenance procedure, firstly, we calculate the received dose of the maintenance personnel when he passes through neighboring grids.

When the maintenance personnel moves from the grid $i$ to its neighboring grid $j$, the received radiation dose is described in (1).

(1) If there are no accessible grids for the ant, it dies and is marked with a no available path mark.

(2) If the ant has reached its targeting grid $\left(V_{\mathrm{o}}\right.$ or $\left.V_{\mathrm{t}}\right)$, it dies and is marked with an available path mark.

(3) Every ant has an original dose $_{k}$ value which equals the maximum allowable radiation dose the maintenance personnel can bear. The value of dose $_{k}$ will reduce gradually when the ant moves in the grids with radiation. The ant will die when the value of $\operatorname{dose}_{k}$ is decreased to zero and it is marked with a no available path mark.

(4) When two ants meet, if the targeting gird of an ant is the same with the original grid of another, the ant having shorter path gives its path data to the other, and then the ant with completed path dies and is marked with an available path mark.

Every ant moves according to (3) and is marked with dead or not and the procedure is repeated until every ant is dead. We call this an iteration calculation step. The maximum allowable amount of the iteration calculation step in the system is iter $_{\max }$.

\section{4) Updating of the pheromone trail}

When one iteration step is finished, all the found maintenance paths are compared and the one with smallest cumulative radiation dose value is selected as the optimal path of this iteration step. The pheromone matrix is undated according to (4).

$$
\tau_{i j}(n+1)=(1-\rho) \tau_{i j}(n)+\Delta \tau_{i j}(n)
$$

$$
\begin{gathered}
\Delta \tau_{i j} \\
=\left\{\begin{array}{l}
\frac{Q}{\sum \operatorname{dose}(i, j)}, \text { if } i, j \text { are in the optimal path } \\
0, \quad \text { if } i, j \text { are not in the optimal path }
\end{array}\right.
\end{gathered}
$$

The formula (4) describes the pheromone relationship between the iteration step $(n+1)$ and the iteration step $n$ of the ant colony algorithm. The parameter $\rho$ is the volatile factor of the pheromone. The parameter $\Delta \tau_{i j}$ denotes the increase quantity of the pheromone after each iteration calculation step and its calculation method is showed in (5). 


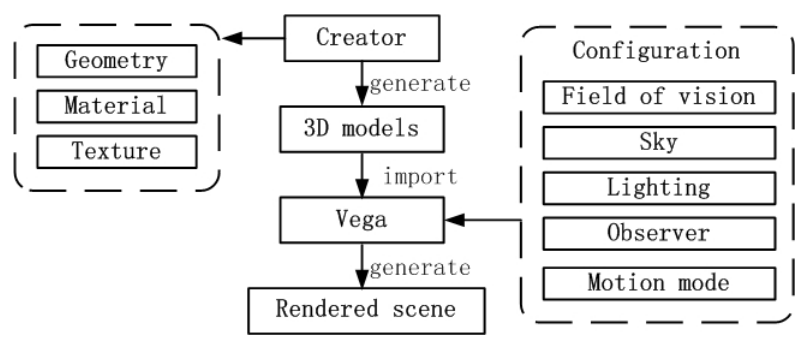

Figure 2. Modeling and rendering procedure

The formula (5) is constructed based on MMAS strategy [16], only if the grids $i$ and $j$ are in the optimal path calculated by the iteration step $n$, their corresponding pheromone increase the value of $\frac{Q}{\sum \operatorname{dose}(i, j)}$.

\section{5) Obtaining of the optimal path}

Each iteration calculation step obtains an optimal path and then the pheromone matrix is updated. Then the new iteration calculation step begins. The procedure is repeated until the iteration step number reaches iter $_{\text {max }}$.

From all the optimal paths calculated by each iteration step, the best one is selected and stored in a file. And this file is used in visualizing of the maintenance path and the driving of the virtual maintenance personnel in the system.

\section{SCENE VISUALIZING}

\section{A. Static scene visualizing}

The nuclear power station includes various equipments, pipes, rooms, etc. At first, these objects were modeled by the Multigen Creator software - a kind of 3D modeling tool. Then these models are organized together to form a static $3 \mathrm{D}$ scene.

Through the 3D rendering engine, the scene can be rendered in a $3 \mathrm{D}$ manner. Users can operate the objects in the nuclear power station scene, and can roam in the scene and get a vivid experience. We use the Multigen Vega graphics engine to render the nuclear power station scene. The modeling and rendering procedure of the nuclear power station is showed in Fig. 2.

\section{B. Radiation dose field visualizing}

Radiation fields are invisible. To show it in the maintenance environment, we use different color planes to represent different radiation dose values. For example, the red color represents the highest radiation dose value and the blue color represents the lowest radiation dose value. And the rendered color radiation environment can help the maintenance personnel to keep away from the dangerous region.

The main rendering process of the radiation field is as follows [10].

1) Read the radiation dose data in the database. The data's form is $(x, y, z, i)$ where $x, y, z$ represents the space position of the sampling point of the radiation field. The parameter $i$ represents the radiation dose value at the sampling point and its value range is $\left[i_{\min }, i_{\max }\right]$.

2) Construct the color array color $[3 n]=\{\{R, G, B\}\}$. With the cut down of the array index $i$, the red color component's values cut down gradually and the blue color component's values raise gradually. The value of parameter $n$ equals to 256 .

3) Do mapping between the radiation dose value and the rendered color. According to the value of the radiation dose at current position, its corresponding color index is calculated. The formula (6) shows the calculation method where the parameter $k_{i}$ denotes the color index of the color array.

$$
k_{i}= \begin{cases}\left\lfloor\frac{i-i_{\min }}{i_{\max }-i_{\min }} \times 3 n\right\rfloor, & i_{\min } \leq i<i_{\max } \\ 3 n-1, & i=i_{\max }\end{cases}
$$

4) Draw the color planes in line with their vertexes' color values color $\left[k_{i}\right]$.

\section{Virtual maintenance personnel visualizing and driving}

We firstly build a virtual maintenance personnel model based on the Multigen Creator tool and some existing virtual people models. Then the behaviors of the virtual maintenance personnel are classified into several types. Then these behaviors are decomposed into some basic actions. These basic action animations are realized through controls to the degree of freedom (DOF) nodes of the virtual maintenance personnel model.

After getting the optimal maintenance path data, the system changes the virtual personnel's positions at regular time in accordance with the key points' coordinates of the path. Besides this, the optimal maintenance path is also drawn in the scene.

\section{IMPLEMENTATION OF THE ALGORITHM AND THE 3D SIMULATION RESULTS}

\section{A. Parameter setting}

Different parameter values have different influence to the stability and convergence ability of the path planning algorithm. Based on the convergence proof given by Stützle et.al. [14] and the experiment results in our system, the parameters' values showed in table I are used.

\section{B. Simulation results}

The rendering results of the nuclear power plant and the radiation field are showed in Fig. 3 and Fig. 4. The path planning result of a maintenance task in radiation environment is showed in Fig. 5. The dash line represents the optimized maintenance path. Fig. 6 shows the virtual maintenance personnel which is walking along the optimal maintenance path.

TABLE I. PARAMETERS VALUE OF THE PATH PLANNING ALGORITHM

\begin{tabular}{|c|c|}
\hline parameters & value \\
\hline$\alpha$ & 1 \\
\hline$\beta$ & 1 \\
\hline$\rho$ & 0.3 \\
\hline$m$ & 16 \\
\hline$Q$ & 5 \\
\hline iter $_{\max }$ & 150 \\
\hline
\end{tabular}


PAPER

Path Planning and Visualizing for Maintenance Simulation of Nuclear Power Plant

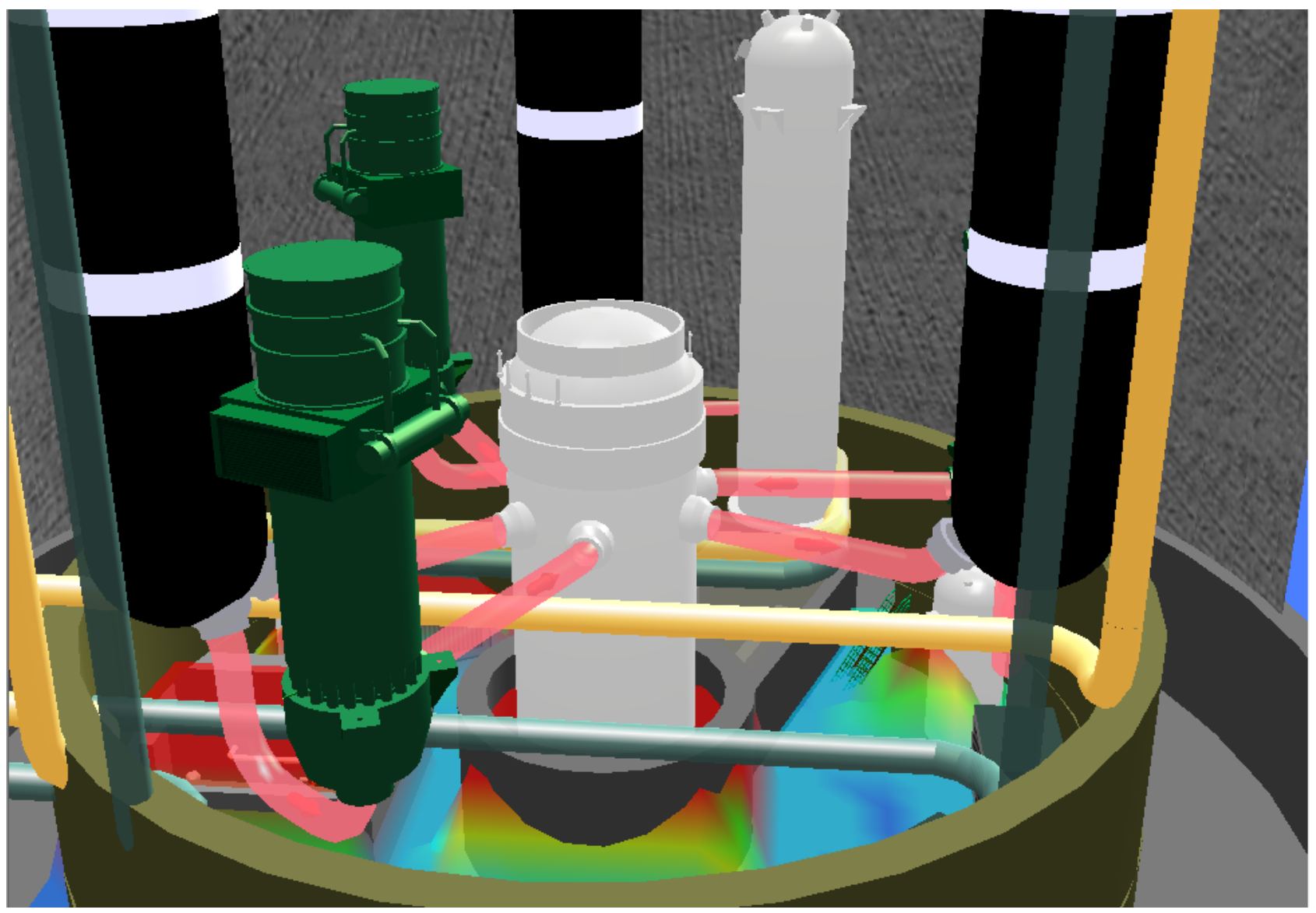

Figure 3. Nuclear power plant scene I

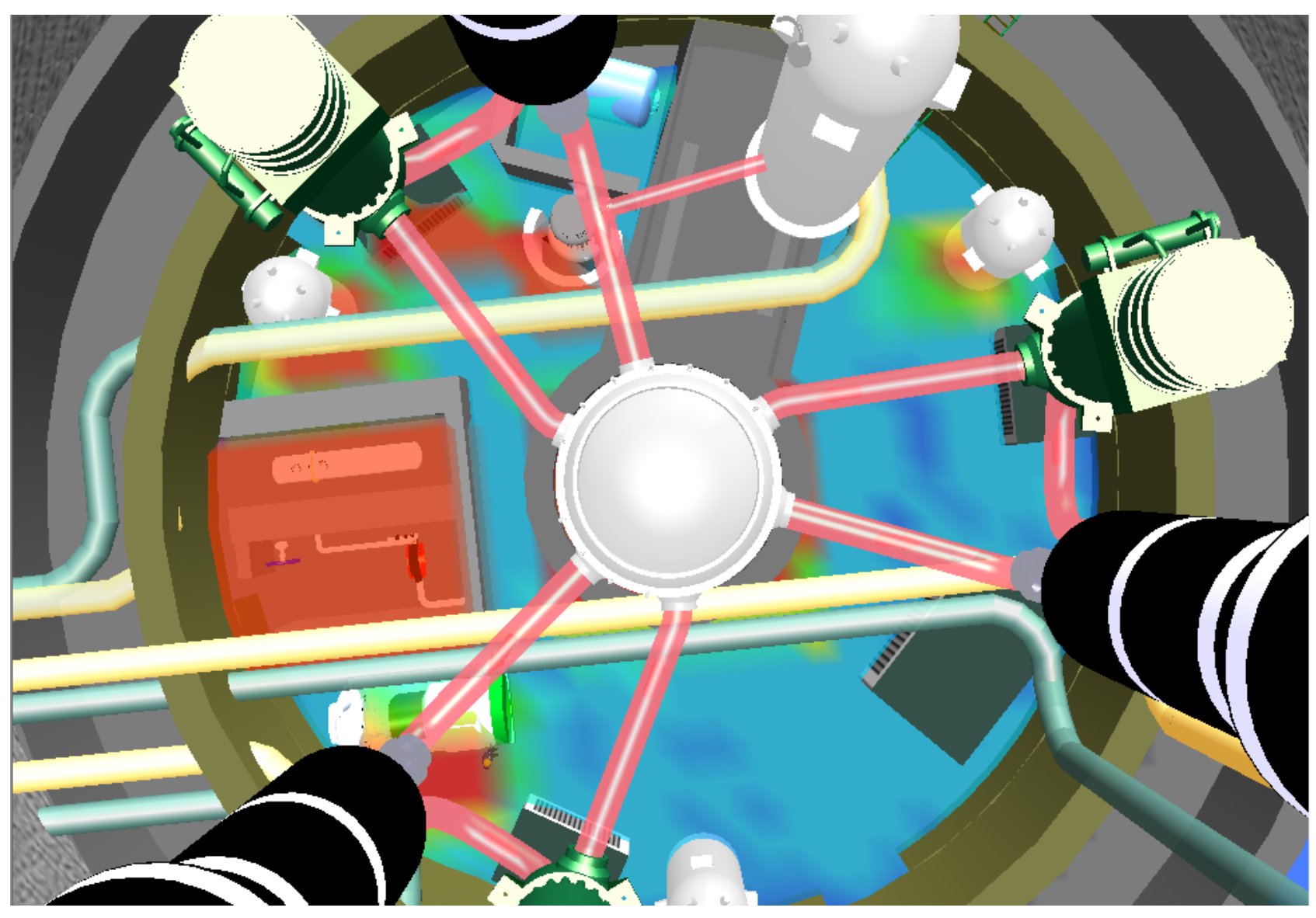

Figure 4. Nuclear power plant scene II 
PAPER

Path Planning and Visualizing for Maintenance Simulation of Nuclear Power Plant

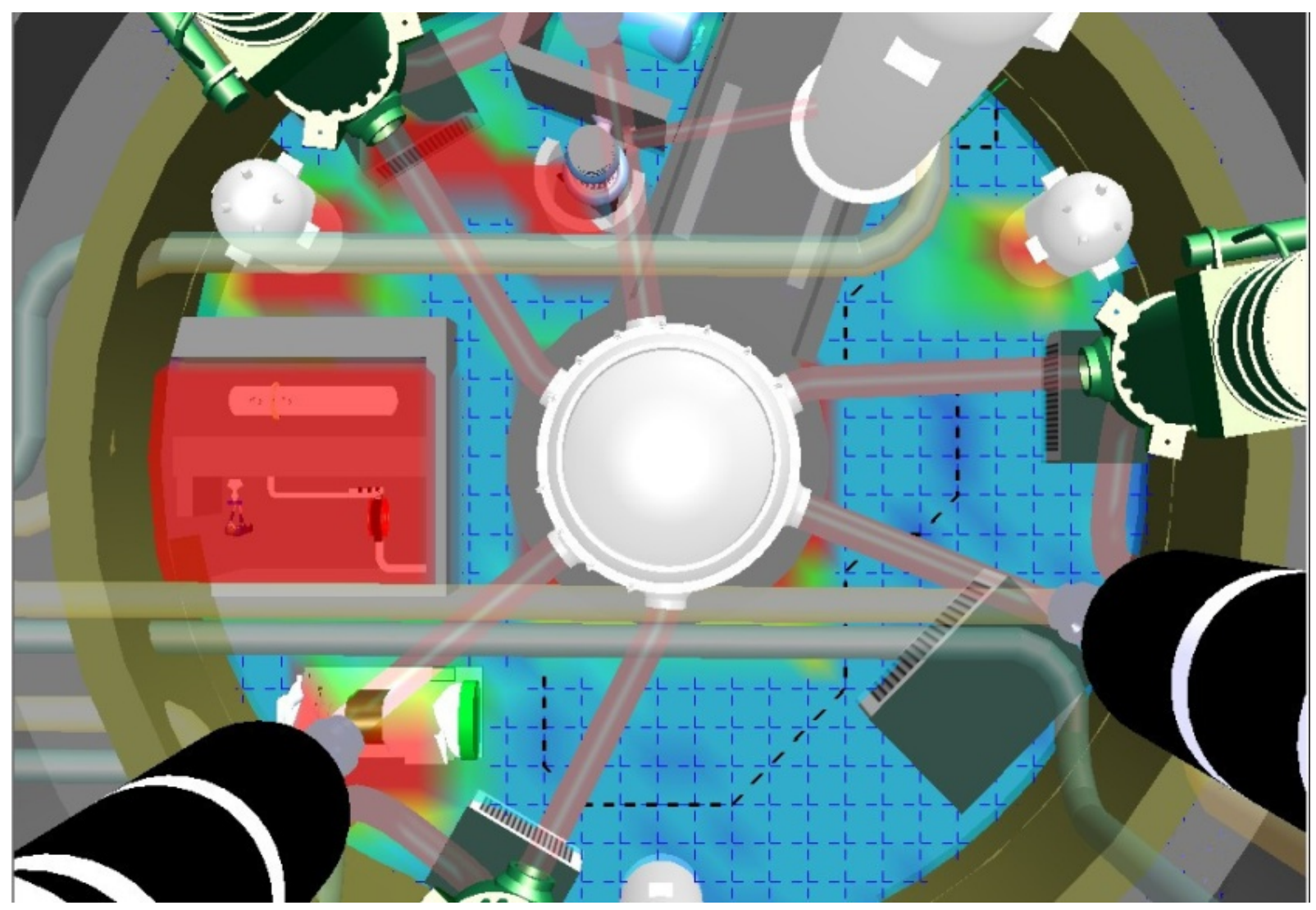

Figure 5. Path planning result

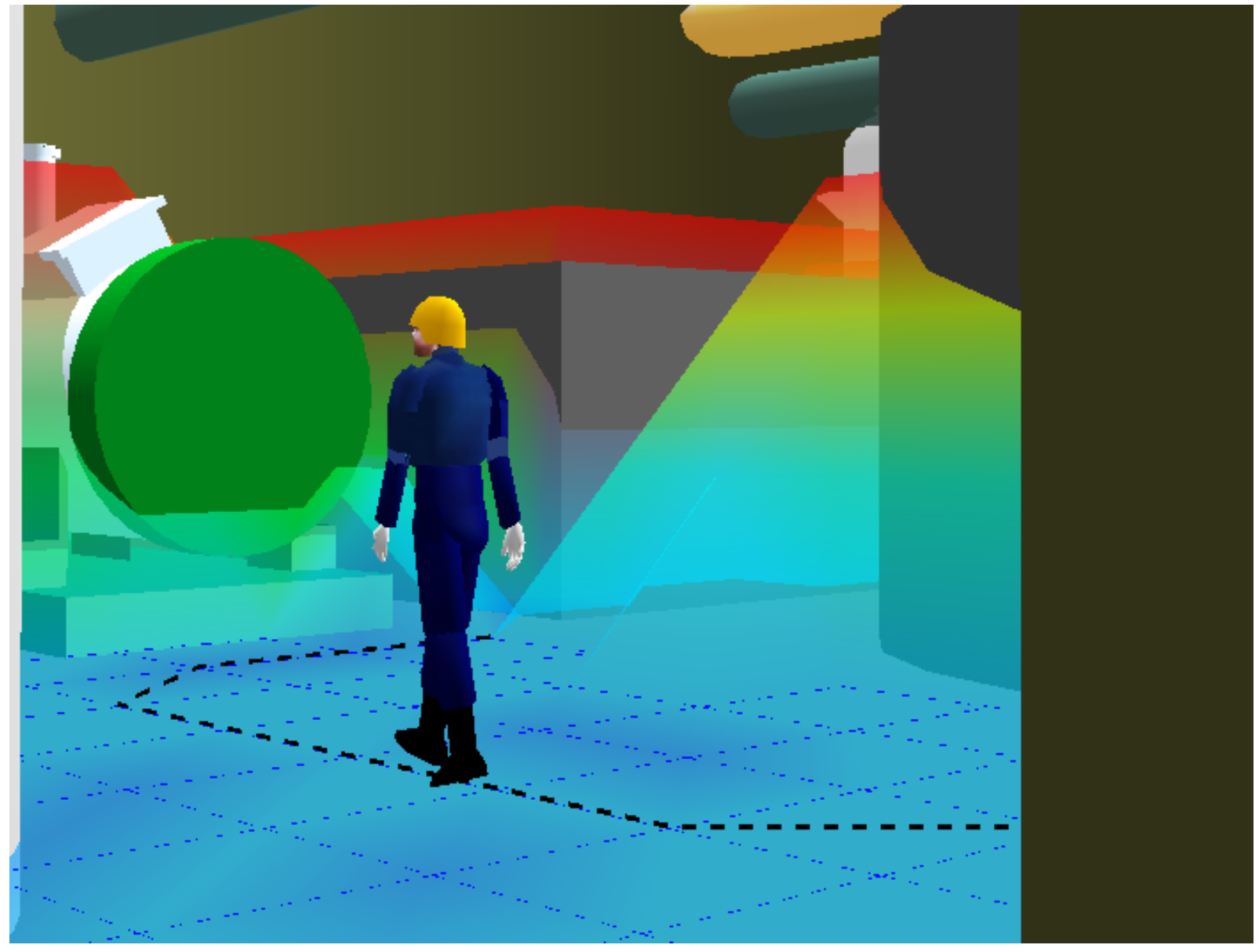

Figure 6. Walking of the virtual maintenance personnel 


\section{CONCLUSION}

To help train maintenance personnel in nuclear power stations and decrease the radiation dose they received, a maintenance path planning method is proposed and a $3 \mathrm{D}$ nuclear power station scene under radiation environment is built. This system can calculate the optimal maintenance path according to the distribution of the radiation field and can provide a vivid virtual operation environment. The system can help increase the safety protection ability of the maintenance personnel in nuclear power stations.

\section{REFERENCES}

[1] Y. Ohga, M. Fukuda, K. Shibata, A system for the calculation and visualization of radiation field for maintenance support in nuclear power plants $[\mathrm{J}]$. Radiation protection dosimetry, 2005,116(14):592-596 http://dx.doi.org/10.1093/rpd/nci014

[2] B. Lamela, A. Felipe, M. Sánchez-Mayoral, Applications of Virtual Reality to Radiation [A]. 11th International Congress of the International Radiation Protection Association. Madrid Spain [C], 2004:05-23

[3] J. Ródenas, I. Zarza, M. Burgos, et al., Developing a virtual reality application for training nuclear power plant operators: setting up a database containing dose rates in the refuelling plant $[\mathrm{J}]$. Radiation protection dosimetry, 2004,111(2): 173-180 http://dx.doi.org/ 10.1093/rpd/nch043

[4] F. Vermeersch, ALARA pre-job studies using the VISIPLAN 3D ALARA planning tool [J].Radiation protection dosimetry, 2005,115(1-4):294-297 http://dx.doi.org/10.1093/rpd/nci115

[5] Z. Liu, Y. Liu, H. Zhu, et al., Research on Nuclear Facilities Decommissioning Engineering Support System Framework [A].Power and Energy Engineering Conference (APPEEC), 2010 Asia-Pacific[C]: IEEE, 2010:1-4

[6] Z. Tang, P. Long, S. Huang, et al., Real-time dose assessment and visualization of radiation field for EAST tokamak [J].Fusion Engineering and Design, 2010,85(7): 1591-1594 http://dx.doi.org/10.1016/j.fusengdes.2010.04.054

[7] G. Rindahl, T. Johnsen, F. Øwre, et al., Virtual reality technology and nuclear decommissioning $[\mathrm{A}]$. Proceedings of the International Conference on Safe Decommissioning for Nuclear Activities, Berlin[C], 2002:223-238

[8] Y. Iguchi, Y. Kanehira, TACHIBANA M, et al. Development of Decommissioning Engineering Support System (DEXUS of the Fugen Nuclear Power Station) [J].Journal of Nuclear Science and
Technology, 2004,41(3):367-375 http://dx.doi.org/10.1080/18811 248.2004.9715497

[9] Y. Kim, W. Park, Use of simulation technology for prediction of radiation dose in nuclear power plant [J].Computational and Information Science, 2005:413-418

[10] G. W. Yan, R. H. Wang, M. Li, Virtual Inspection System of Nuclear Power Plants with Nuclear Radiation [J]. International Journal of Digital Content Technology and its Applications, 2012,6(10):385-393

[11] C. Wang, J. Q. Zhou, L. J. Li, Creator Visual Simulation Modeling Technology [M]. Wuhan: Huazhong University of Science \& Technology Press, 2005

[12] Z. R. Gong, Vega Programming [M]. Beijing: National Defence Industry Press, 2002

[13] M. Dorigo, V. Maniezzo , A. Colorni, Ant system: optimization by a colony of cooperating agents [J]. IEEE Transaction on Systems, Man, and Cybernetics-Part B, vol. 26, no. 1, pp. 29-41, 1996 http://dx.doi.org/10.1109/3477.484436

[14] T. Stützle, M. Dorigo, A short convergence proof for a class of ant colony optimization algorithms [J]. IEEE Transactions on Evolutionary Computation, 2002, 6(4):358-365 http://dx.doi.org/10.1109/TEVC.2002.802444

[15] B. Bullnheimer, R. F. Hartl, C. Strauss, An improved ant System algorithm for the vehicle Routing Problem [J]. Annals of Operations Research, 1999, 89:319-328 http://dx.doi.org/ 10.1023/A:1018940026670

[16] T. Stützle, H. H. Hoos, MAX-MIN Ant System [J]. Future Generation Computer Systems, 2000, 16(8):889-914 http://dx.doi.org/10.1016/S0167-739X(00)00043-1

\section{AUTHORS}

Guangwei Yan is with the School of Control and Computer Engineering, North China Electric Power University, Beijing, China. He is an associate professor of this school. He received his $\mathrm{PhD}$ degree in Computer Application Technology from Beijing Institute of Technology, Beijing, China in 2005 (e-mail: yan_guang_wei@126.com).

Miao Li is with the School of Control and Computer Engineering, North China Electric Power University, Beijing, China, 102206 (e-mail: lee_miao@126.com).

Submitted 14 July 2013. Published as re-submitted by the authors 12 October 2013. 